\title{
A NOTE ON SYMMETRIC FUNCTIONS IN FORMANEK POLYNOMIALS
}

\author{
SHMUE L ROSSET
}

\begin{abstract}
Formanek's proof of the existence of central identities has the following form: one constructs $n$ polynomials $G\left(X, Y_{1}, Y_{2}, \cdots, Y_{n}\right)$, $G\left(X, Y_{2}, \cdots, Y_{n}, Y_{1}\right)$, etc., whose sum is the desired central identity. The variables $X, Y_{i}$ are generic matrices. These $G$ 's commute pairwise which raises the question whether all symmetric functions in them also give central identities. Here we show that this is not so for $n>2$, and connect this question with Amitsur's solution of the general crossed product problem.
\end{abstract}

E. Formanek [1] gave a beautiful solution to a problem of Kaplansky. The problem was to find a polynomial $p\left(X_{1}, \cdots, X_{m}\right)$ in free (noncommuting) variables such that for every substitution $X_{i}=a_{i}$, where $a_{i}$ are $n \times n$ matrices, $p\left(a_{1}, \cdots, a_{m}\right)$ is a central matrix, and not always zero. Such $p(X)$ is called a central identity.

Formanek's ingenious solution was as follows. Let

$$
\begin{aligned}
g\left(x_{1}, \cdots, x_{n+1}\right) & =\prod_{j=2}^{n}\left(x_{1}-x_{j}\right)\left(x_{n+1}-x_{j}\right) \prod_{2 \leq j<k \leq n}\left(x_{j}-x_{k}\right)^{2} \\
& =\sum_{(a)} c_{(a)} x_{1}^{a_{1}} x_{2}^{a_{2}} \cdots x_{n+1}^{a_{n+1}} .
\end{aligned}
$$

Then let

$$
G\left(X, Y_{1}, \cdots, Y_{n}\right)=\sum_{(a)} c_{(a)} X^{a_{1}} Y_{1} X^{a}{ }^{2} Y_{2} \cdots X^{a_{n}} Y_{n} X^{a_{n}+1}
$$

The sum $G\left(X, Y_{1}, \cdots, Y_{n}\right)+G\left(X, Y_{2}, \cdots, Y_{n}, Y_{1}\right)+\cdots+G\left(X, Y_{1}, Y, \cdots, Y_{n-1}\right)$ is the desired central identity.

The following question now arises: Are all the basic symmetric functions in the expressions comprising the sum also central identities? Here I show that the answer is no. In $\$ 2$ of this note I show what the origin of this question was.

Received by the editors February 19, 1974.

AMS (MOS) subject classifications (1970). Primary 16A38, 16A40.

Key words and phrases. Formanek polynomials, generic matrices, central identities, crossed product. 
In this note some familiarity with [1] is assumed.

1. For briefness of notation let $G\left(X, Y_{1}, \cdots, Y_{n}\right)=G_{1}, G\left(X, Y_{2}, \cdots, Y_{1}\right)$ $=G_{2}$, etc. Let $X=\left(x_{\alpha \beta}\right), Y_{i}=\left(y_{\alpha \beta}^{i}\right)$ where $x_{\alpha \beta}$ and $y_{\alpha \beta}^{i}$ are independent variables. Here $\alpha, \beta, i=1,2, \cdots, n$. Such matrices are called generic matrices for obvious reasons. It is easy to see that $X$ is diagonalizable and the basic computation of [1] shows that for $X$ diagonal $G_{1}, G_{2}, \cdots, G_{n}$ are also diagonal. Thus $X, G_{1}, \cdots, G_{n}$ can be simultaneously diagonalized and therefore they commute with each other. Let $s_{1}, s_{2}, \cdots, s_{n}$ be the basic symmetric functions. Thus $s_{1}\left(x_{1}, \cdots, x_{n}\right)=\Sigma x_{i}, s_{n}\left(x_{1}, \cdots, x_{n}\right)=x_{1} \cdots$ $x_{n}$, etc. Suppose $s_{j}\left(G_{1}, \cdots, G_{n}\right)$ are central identities for $j=1,2, \cdots, n$. The symmetric functions $p_{l}\left(x_{1}, \cdots, x_{n}\right)=\sum_{j=1}^{n}\left(x_{j}\right)^{l}, l=1,2, \cdots, n$ can be expressed as integral polynomials of $s_{1}, \cdots, s_{n}$. Hence $p_{l}\left(G_{1}, \cdots, G_{n}\right)$ would also be central for $l=1,2, \cdots, n$. Now we show that this leads to contradiction in two ways, the second of which is less general, but rather illuminating, and is in $\$ 2$.

To see that $p_{j}\left(G_{1}, \cdots, G_{n}\right)$ cannot be central for all $j=1,2, \cdots, n$ it is enough to take $X$ to be diagonal. Thus let $X=\left(x_{i} \delta_{i j}\right)$, where $x_{1}, \cdots, x_{n}$ are indeterminates. As remarked above, for $X$ diagonal all $G_{i}$ are diagonal. Thus

$$
G_{j}=\left(\begin{array}{ccc}
b_{j 1} & & \\
& \cdot & 0 \\
0 & \cdot & \\
& & b_{j n}
\end{array}\right)
$$

where $h_{j k}$ is a polynomial in the variables $x_{i}, y_{\alpha \beta}^{i}$ and $i, j, \alpha, \beta=1,2$, $\cdots, n$. The polynomials $h_{j k}$ are all distinct and nonzero. Indeed, one can find a substitution, as in [1], such that $h_{j k} \neq 0$ and $h_{j l}=0$ for $l \neq k$. Actually it is easy to show that each $h_{j k}$ is the product of $g\left(x_{1}, \cdots, x_{n}, x_{1}\right)$ with some homogeneous polynomial in the $y-s$. But this is of no use to us here.

If $\sum_{i=1}^{n}\left(G_{i}\right)^{l}$ is a central identity this implies that $\sum_{j=1}^{n}\left(h_{j k}\right)^{l}=H_{l}$ is constant, i.e. $H_{l}$ does not depend on $k$, for all $k=1,2, \cdots, n$. For example $\sum_{j=1}^{n}\left(h_{j 1}\right)^{l}=\sum_{j=1}^{n}\left(h_{j 2}\right)^{l}$ for $l=1,2, \cdots, n$. As no two $h_{j k}$ can be equal for a fixed $j$ this implies that $\left(h_{12}, \cdots, h_{n 2}\right)$ is a permutation of $\left(h_{11}, \cdots, h_{n 1}\right)$, and in general each $\left(h_{1 k}, \cdots, h_{n k}\right)$ is a permutation of $\left(h_{11}, \cdots, h_{n 1}\right)$.

Let $Z_{i}=\left(z_{\alpha \beta}^{i}\right)$ be another set of $n$ generic $n \times n$ matrices. Thus $\left\{z_{\alpha \beta}^{i}\right\}$ 
is a new set of variables independent of the $x-s$ and $y-s$. Certainly $G_{j}\left(X, Z_{1}, \cdots, Z_{n}\right)$ is obtained from $G_{j}\left(X, Y_{1}, \cdots, Y_{n}\right)$ by replacing $y_{a \beta}^{i}$ by $z_{a \beta}^{i}$ It follows now that the sum

$$
\sum_{j=1}^{n} G_{j}\left(X, Y_{1}, \cdots, Y_{n}\right) G_{j}\left(X, Z_{1}, \cdots, Z_{n}\right)
$$

is a central identity. For it is equal to the diagonal matrix with $(k, k)$ th entry which is $\sum_{j=1}^{n} h_{j k}(x, y) h_{j k}(x, z)$ and this is independent of $k$ as follows from the considerations above. Now we have our contradiction, since the sum $\sum_{i=1}^{n} G_{i}(X, Y) G_{i}(X, Z)$ is not a central identity. Indeed, let $Y_{1}=e_{12}, Y_{2}=$ $e_{23}, \cdots, Y_{n-1}=e_{n-1, n}, Y_{n}=e_{n 1}$ and $Z_{1}=e_{13}, Z_{2}=e_{32}, Z_{3}=e_{24}, \cdots$, $Z_{n}=e_{n 1}$. Here $e_{i j}$ is the matrix which has 1 in position $(i, j)$ and 0 elsewhere. This substitution gives

$$
\begin{aligned}
& G_{1}(X, Y)=g(x) e_{11}, \quad G_{2}(X, Y)=g(x) e_{22}, \cdots, \quad G_{n}(X, Y)=g(x) e_{n n}, \\
& G_{1}(X, Z)=g(x) e_{11}, \quad G_{2}(X, Z)=g(x) e_{33}, \cdots, \quad G_{n}(X, Z)=g(x) e_{n n} .
\end{aligned}
$$

Thus $\sum_{j=1}^{n} G_{j}(X, Y) G_{j}(X, Z)$ is not 0 but has 0 in the $(2,2)$ position and therefore is not central.

To sum up this section we make a formal statement.

Theorem. Let $s_{1}, \cdots, s_{n}$ be the basic symmetric polynomials. Then $s_{1}\left(G_{1}, \cdots, G_{n}\right), \cdots, s_{n}\left(G_{1}, \cdots, G_{n}\right)$ are not all central identities.

2. Amitsur [2] proved that the following division algebra is not a crossed product. Let $n$ be an integer divisible by $p^{2}$, where $p$ is an odd prime, or by 8. Let $F$ be a field of characteristic 0 , and let $X_{1}, \cdots, X_{m}, m \geq 2$, be generic $n \times n$ matrices. Then the ring $R=F\left[X_{1}, \cdots, X_{m}\right]$ is a domain and it has a total ring of fractions $D$ which is a division ring of dimension $n$ over its center $K$. This $D$ was Amitsur's example. In our case $R=F\left[X, Y_{1}, \cdots, Y_{n}\right]$ with $D, K$ having the analogous meaning.

Now suppose the Theorem proved is false. This would mean that the polynomial $p(t)=\left(t-G_{1}\right)\left(t-G_{2}\right) \cdots\left(t-G_{n}\right)$ has coefficients in $K$. Furthermore, it is irreducible because it is clear that no sum $G_{i_{1}}+\cdots+G_{i_{r}}$, where $\left(i_{1}, \cdots, i_{r}\right)$ is a proper nonempty subset of $(1,2, \cdots, n)$, is a central identity. Thus the field $K\left(G_{1}, \cdots, G_{n}\right)$ is a Galois extension, even a cyclic extension of $K$, of degree $n$ over $K$ and hence a maximal subfield of $D$. This implies that $D$ is cyclic, which is absurd. 
We end by remarking that perhaps if $n$ is prime one might find a polynomial, using Formanek's trick, such that the symmetric.functions are central. This, of course, will resolve the part of the crossed product problem still open.

\section{REFERENCES}

1. E. Formanek, Central identities for matrix rings, J. Algebra 23 (1972), 129132.

2. S. A. Amitsur, On central division algebras, Israel J. Math. 12 (1972), 408420. MR $47 \# 6763$.

DEPARTMENT OF MATHEMATICAL SCIENCES, TEL-AVIV UNIVERSITY, TEL-AVIV, ISRAEL 